\title{
Attenuation of acute systemic inflammatory response after valve surgery
}

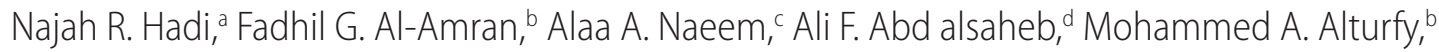

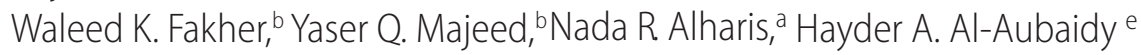

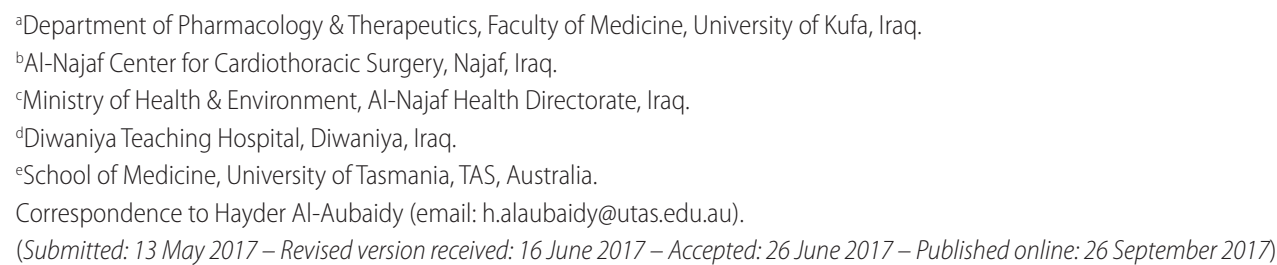

\begin{abstract}
Objective This study highlights the protective effects of montelukast on myocardial ischemic reperfusion injury induced by cardiopulmonary bypass during valve replacement surgery.

Methods A total of 60 patients with valvular disease undergoing elective valve surgery were enrolled in this randomized single-blinded study. Participants were divided into two main groups: Montelukast-treated group consisted of 30 patients who were given 10 mg montelukast sodium (Singulair ${ }^{\oplus}$, MSD, USA) tablet, once daily at bedtime for 3 days before valve surgery. Control group consisted of 30 patients who underwent valve surgery without taking montelukast tablets. Blood samples were collected at following times $\left(T_{0^{\prime}} T_{1}\right.$ before aortic cross clamp; $T_{2}$ after aortic cross clamp; and $\mathrm{T}_{3} 24 \mathrm{~h}$ after the surgery), for measuring several inflammatory markers. Ejection fraction (EF) was measured before surgery and three months after surgery. Pulmonary functions were measured before and after the surgery in both study groups.

Results There were significant increase in the levels of TNF- $\alpha, I L-6, \alpha 2$ macroglobulin/creatinine ratio and CTnl, in the control group compared to the montelukast-treated group among different study times, $(P<0.05)$. In addition, the EF was significantly higher in the montelukast-treated group after the valve surgery, $(P<0.05)$. Levels of forced vital capacity (FVC), forced expiratory volume $1\left(F E V_{1}\right)$, and $\mathrm{FEV}_{1} / \mathrm{FVC}$ ratio where significantly higher in the montelukast-treated group than the control group, $(P<0.05)$.

Conclusion This study shows the benefits of using pre-surgical montelukast supplement in ameliorating the inflammatory process in patients undergoing cardiopulmonary bypass during valve replacement surgery.

Keywords montelukast, mitral and aortic valve replacement surgery, ischemia reperfusion injury, interleukin-6, cardiac troponin 1, tumor necrotic factor-alpha, alpha 2 macroglobulin/creatinine, ejection fraction, forced vital capacity (FVC), forced expiratory volume in one second $\left(F E V_{1}\right), F_{1}, / F V C$ ratio
\end{abstract}

\section{Introduction}

Cardiopulmonary bypass (CPB) is an important procedure, which is routinely performed as part of a classical cardiac surgery and support of life, ${ }^{1}$ but it is associated with injury that may induce pathological changes in the form of systemic inflammatory response syndrome (SIRS) or multiple organ dysfunction syndrome (MODS). ${ }^{2}$ SIRS remains as a distress in open-heart surgery, and lack of adequate patient screening is an ongoing problem. ${ }^{3,4}$ It is particularly noteworthy that SIRS is initiated by many factors including surgical trauma, reperfusion of ischemic organ and CPB. ${ }^{4}$ The $\mathrm{CPB}$ is the major factor for initiating SIRS since off-pump cardiac surgery has been shown to significantly reduce inflammatory response. ${ }^{5,6}$ Other related factors triggering the inflammatory response including hemodilution; electrolyte imbalance; pharmacological agents which are used during surgery; myocardial cardioplegic arrest; formation of heparinprotamine complex; and the release of endothelin and the expression of adhesion molecule on leukocyte and endothelium. ${ }^{5,6}$ There are evidences supporting that $\mathrm{CPB}$ can induce the activation of most of the body's major host defensive processes, include the activation of various complements, coagulation factors, kinins, fibrinolysis, leukocytes, platelets and inflammatory cytokines. This study aims to evaluate the possible protective effects of montelukast as a selective cystienyl leukotriene-1 receptor antagonist in the myocardial ischemic reperfusion injury induced by heart valve replacement surgery.

\section{Patients and Methods}

Sixty patients (23 males and 37 females) with valvular heart disease undergoing elective valve surgery were randomly included in this single-blinded clinical trial. The study was conducted at the Cardiothoracic Center, Al Sadir Medical City, Najaf, Iraq between April 2015 and December 2016. Participants were classified into two main study groups:

\section{Group 1}

Montelukast-treated group (MK group) included 30 patients who underwent valve surgery (14 patients had a mitral valve replacement, MVR) and (16 patients had an aortic valve replacement, AVR). Participants in this group were given $10 \mathrm{mg}$ montelukast sodium (Singulair ${ }^{\circ}$, MSD, USA) tablet, once daily at bed time for 3 days before valve surgery. ${ }^{8,9}$

\section{Group 2}

(Control group, C group) included 30 participants who underwent valve replacement surgery (17 patients had a mitral valve replacement, MVR) and (13 patients had an aortic valve replacement, AVR). Participants in this group had no montelukast supplement, and they were considered as controls.

Blood samples were collected at following times, ( $T_{0}$ after anesthesia; $T_{1}$ before aortic cross clamp; $T_{2}$ after aortic cross clamp; $T_{3} 24 \mathrm{~h}$ after the valve replacement 
surgery), for the measurements of the following inflammatory biomarkers (tumer necrotic factor alpha (TNF- $\alpha$ ); Interleukin 6 (IL-6); Alpha 2 macroglobumin over creatinine ( $\alpha 2$ macroglobulin/creatinine) ratio and cardiac troponin 1 (cTnI). For all participants, serum samples were separated and stored at $-80^{\circ} \mathrm{C}$ until analysis. The determination of cytokines was formed using an ELISA assay (Cloud-Clone Corp., USA).

Bronchial wash was collected from each participants before the valve replacement surgery and after the completion of the operation in order to determine the levels of IL6 and TNF $\alpha$.

Echocardiography was also performed to determine the ejection fraction (EF) before the surgery and was repeated three months after the surgery.

Pulmonary function test was undertaken 2 days before the surgery and 10 days after the surgery in order to evaluate the lung protective effects of montelukast in non-asthmatic participants to determine the levels of the forced vital capacity (FVC); forced expiratory volume $1\left(\mathrm{FEV}_{1}\right)$; and the $\mathrm{FEV}_{1} / \mathrm{FVC}$ ratio as measured by Spirometer.

\section{Statistical Analysis}

Data were analyzed to determine the mean and standard error of the mean using statistical package for social sciences (SPSS) version 20 software for windows. The study used independent sample $t$-test, paired $t$-test and chi-squared test for categorical variables. A $P$-value of $\leq 0.05$ was considered significant.

\section{Results}

Participants of the two study groups were comparable for age, gender and comorbidities (Table 1).

\section{Tumor necrotic Factor Alpha (TNF- $\alpha$ ) Concentration}

Serum levels of TNF- $\alpha$ were significantly lower in the montelukast-treated group compared to the control group,

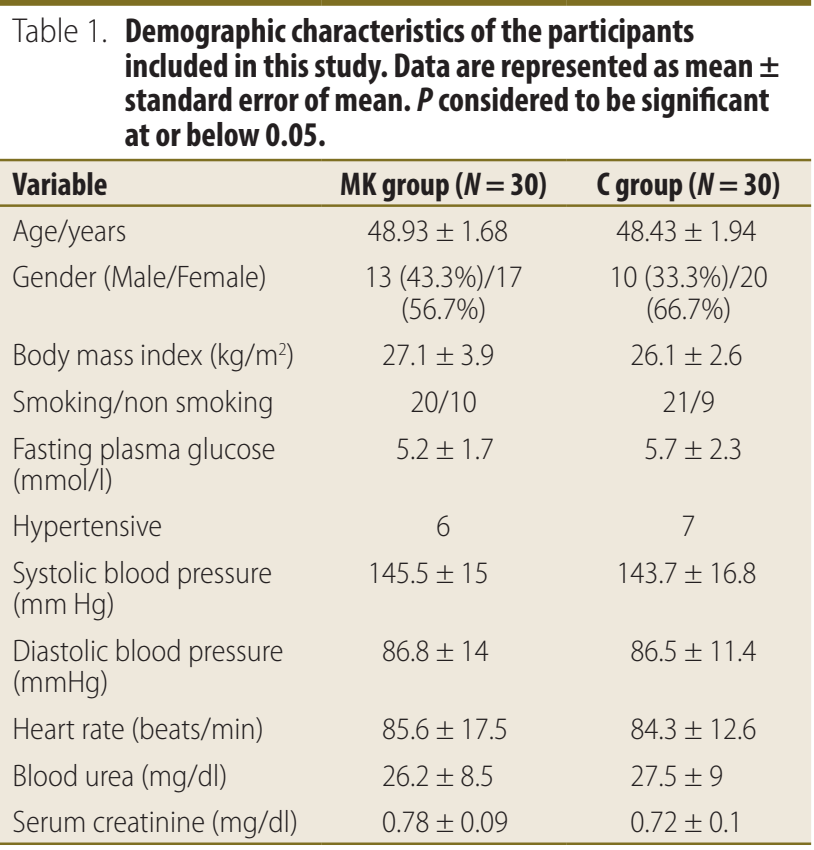

* significant difference between the two study groups at any time at $P$ equal or less than 0.050
$(P<0.05)$, at different study times: before anesthesia; before aortic cross clamp; after aortic cross clamp and $24 \mathrm{~h}$ after surgery as shown (Fig. 1).

\section{Interleukine 6 (IL6) Concentration}

The IL-6 levels were significantly $(P<0.05)$ lower in montelukast-treated group as compared to the control group at all study times (Fig. 2).

\section{Cardiac Troponin I (cTnl)}

The concentrations of cTnI were significantly lower in the montelukast-treated group compared to the control group at the all selected study times, $(P<0.05)$, (Fig. 3 ).

\section{Alpha 2 Macroglobulin/Creatinine Ratio (A2M/Cr)}

Similarly, the levels of the $\alpha 2 \mathrm{M} / \mathrm{Cr}$ ratio were significantly $(P<0.05)$ lower in the montelukast-treated groupy times (Fig. 4).

\section{Ejection Fraction (EF)}

The EF was significantly higher in the montelukast-treated group compared to the Control group after the valve replacement surgery, $(P<0.05)$, (Fig. 5).

\section{FEV /FVC Ratio}

This study showed a non-significant difference in $\mathrm{FEV} / \mathrm{FVC}$ ratio between the two study groups before the valve replacement surgery. However, the ratio became statistically significant, $(P<0.05)$, in the montelukast-treated group as compared to the Control group (Fig. 6).

\section{Correlation Between IL-6 and FEV/FVC Ratio}

There was a significant negative correlation between the IL-6 in the bronchial wash and FEV1/FVC ratio, $(r=-0.675$, $P<0.001$ ), for all participants (Fig. 7).

\section{Correlation Between TNF- $\alpha$ and FEV/FVC Ratio}

Similarly, these was a significant negative correlation between the levels of TNF- $\alpha$ in bronchial wash and the $\mathrm{FEV}_{1} / \mathrm{FVC}$ ratio, ( $r=-0.708, P<0.001)$, (Fig. 8).

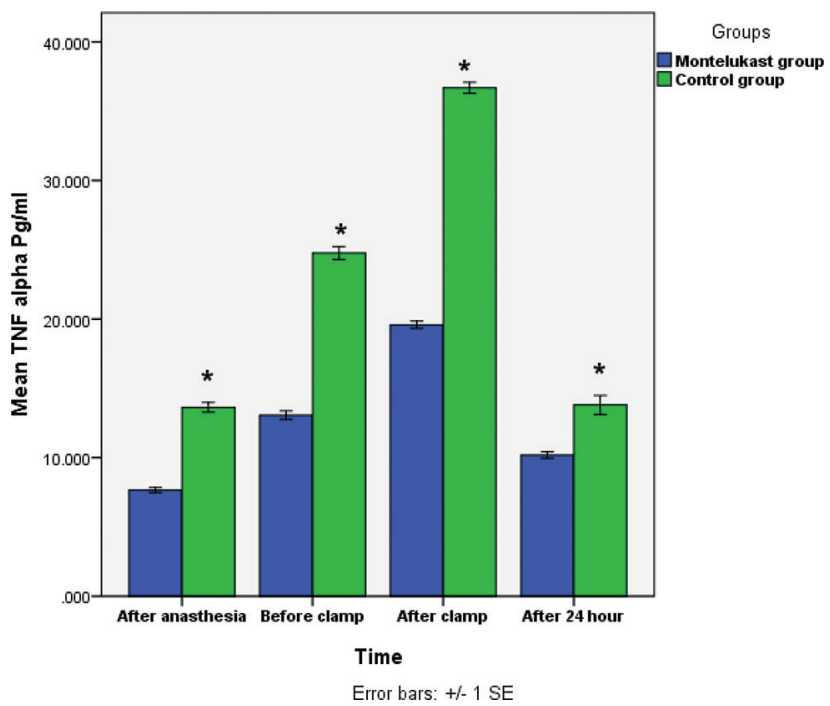

Fig. 1 TNF- $\alpha$ concentration at different study times into two groups; montelukast-treated group and control group. 


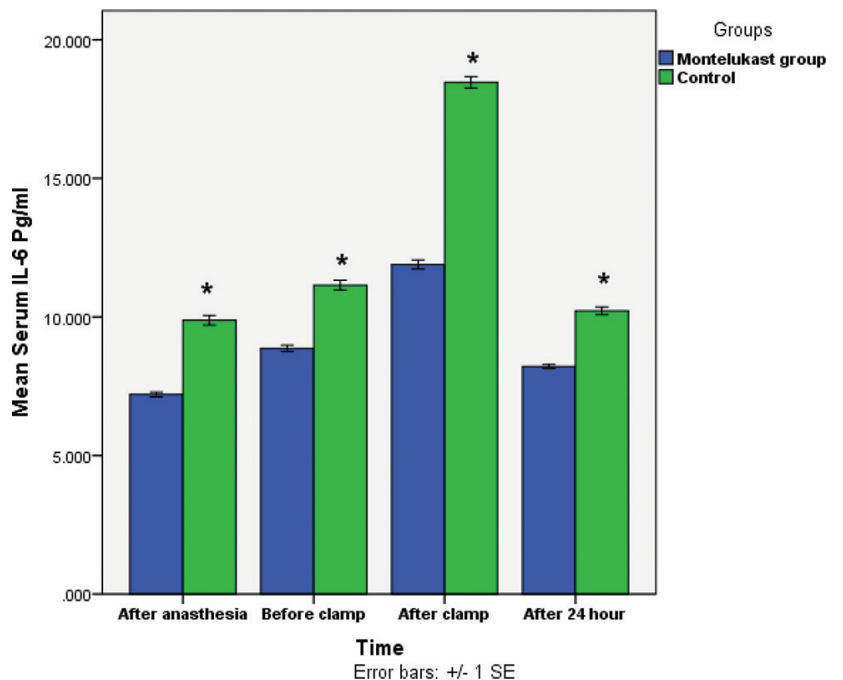

Fig. 2 IL-6 concentration at different study times into two groups; montelukast-treated group and control group.

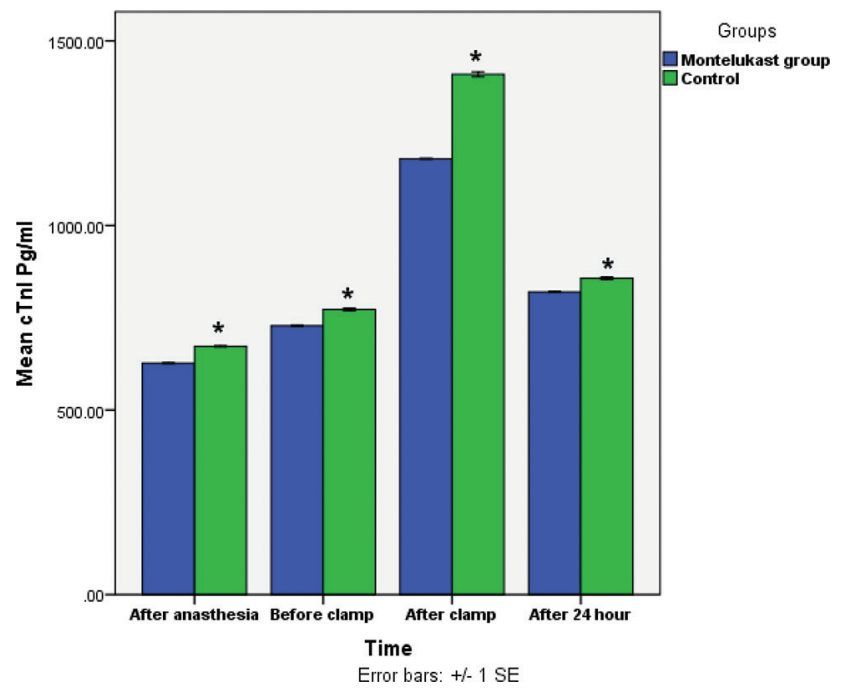

Fig. 3 CTnl concentration at different study times into two groups; montelukast-treated group and control group.

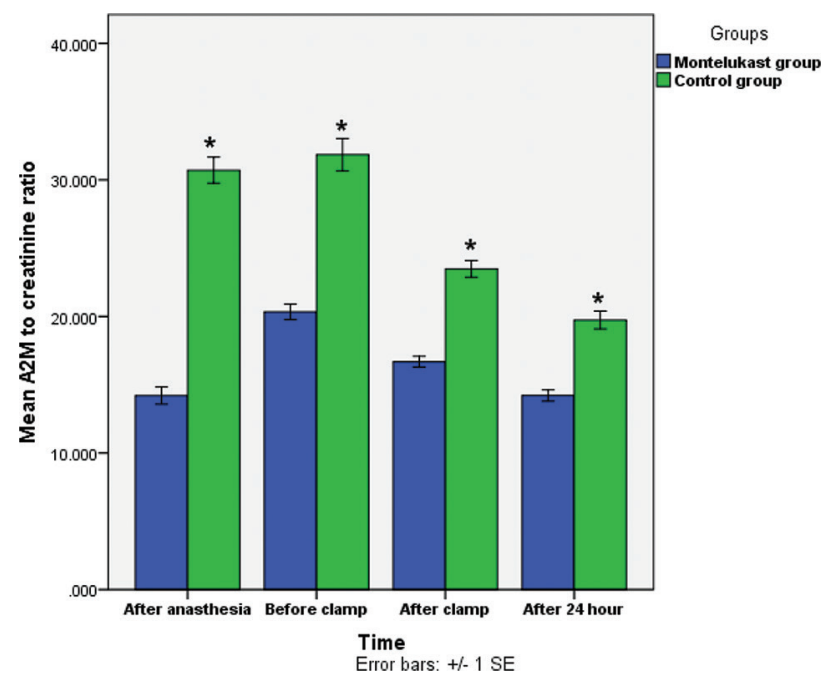

Fig. $4 \alpha 2 M / C r$ concentration at different study times into two groups; montelukast-treated group and control group.

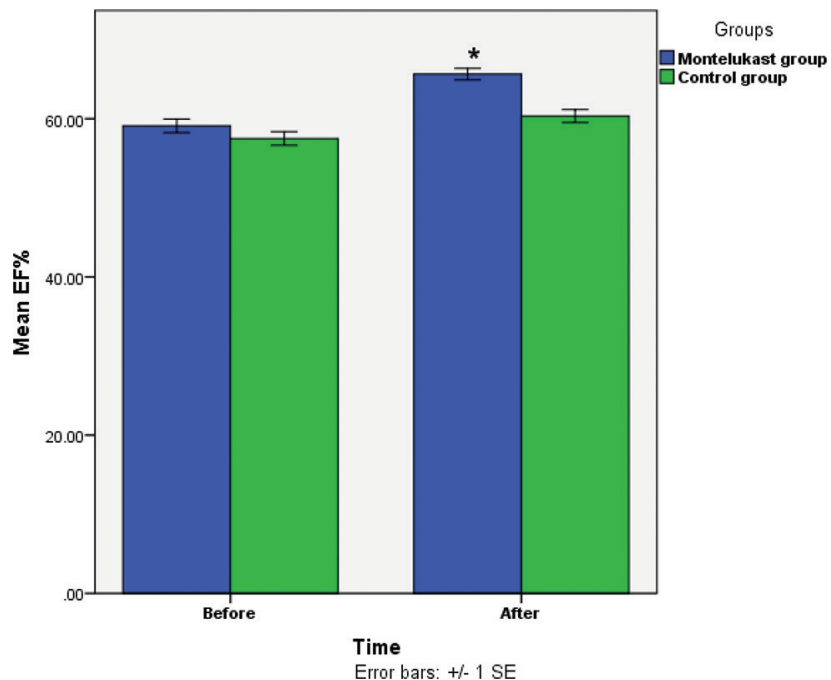

Fig. 5 Ejection fraction before and after valve surgery in montelukast-treated group and control group.

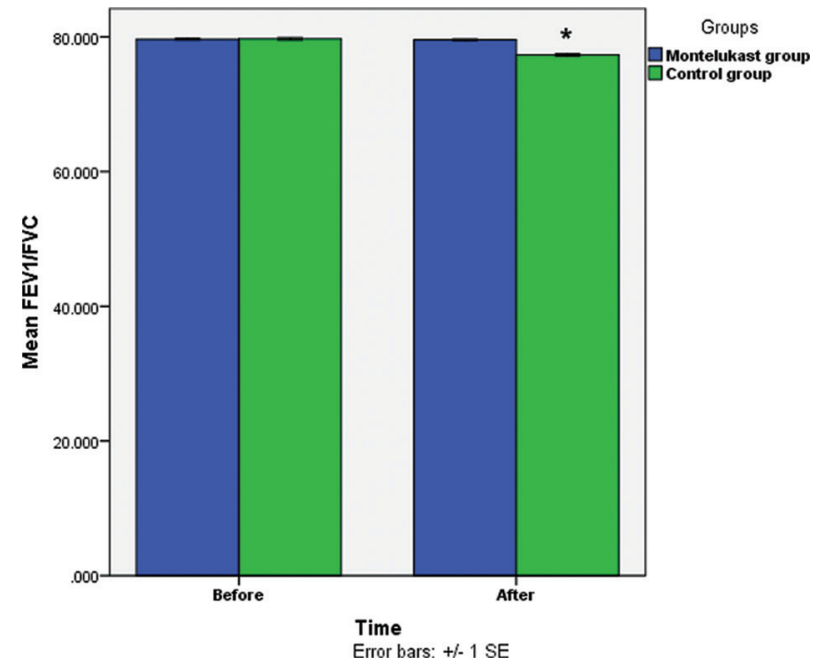

Fig. $6 \mathrm{FEV}_{1} / \mathrm{FVC}$ before and after valve surgery in montelukasttreated group and control group.

\section{Discussion}

Leukotrienes (LTs) are defined as bioactive proinflammatory molecules that are produced by the 5-lipoxygenase pathway from arachidonic acid metabolism in many cells, including epithelial cells, fibroblasts, myoblasts, smooth muscle cells, basophils, eosinophils, neutrophils, macrophages, and lymphocytes. They play potent inflammatory roles in human body response. ${ }^{10}$ These cytokines, particularly TNF- $\alpha$, are early regulators of the immune response and can induce the release of secondary cytokines, such as IL- 6 and TNF- $\alpha$, which provokes neutrophil-mediated tissue injury by acting on endothelial cells and other neutrophils. ${ }^{10}$ This study focussed on the anti-inflammatory effects of specific cytokines, (TNF- $\alpha$ and IL-6), in ameliorating the inflammatory response after elective heart valve surgery. Both levels of these cytokines were significantly lower, (at all times), in the montelukast-treated groups as compared to the Control group, $P<0.05$, (Figs 1 and 2). This proves the importance of using anti-inflammatory 


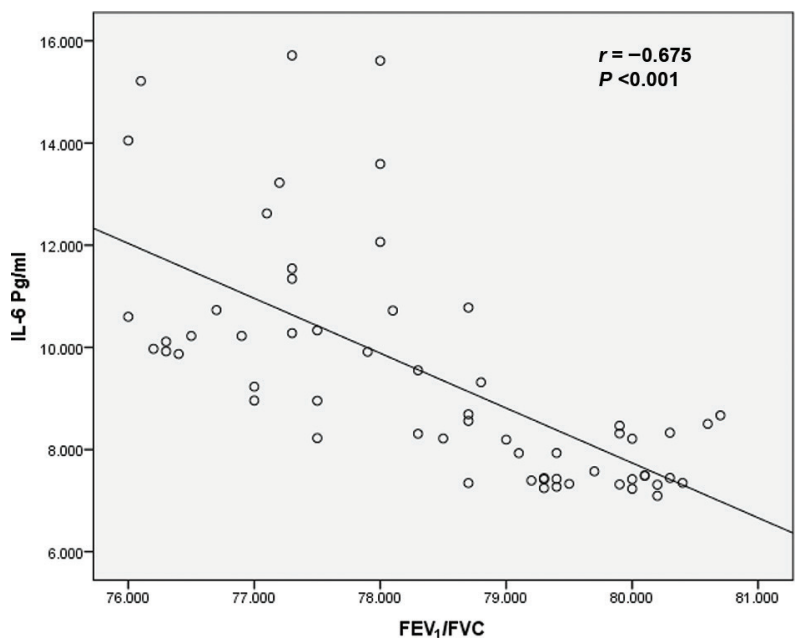

Fig. 7 Correlation between IL- 6 and FEV/FVC.

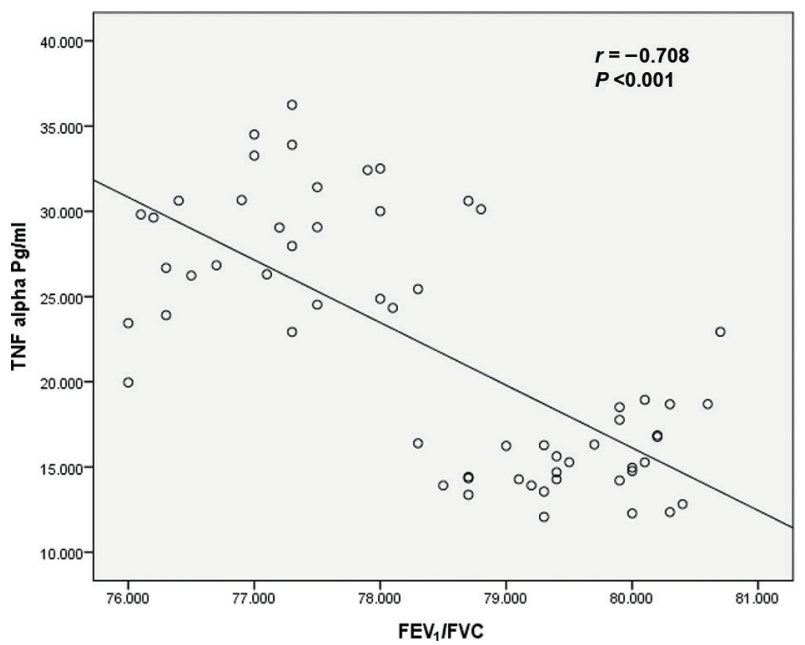

Fig. 8 Correlation between TNF- $\alpha$ and FEV /FVC. supplements prior to any invasive heart surgery to reduce the inflamatory result and improves the outcomes. ${ }^{11,12}$

Our findings also indicated that montelukast is able to reduce the levels of cTnI (Fig. 3). This is in agreement with the previous studies and confirm that montelukast, at high doses, might have cardioprotective effects during endotoxemia attributed to its antioxidant and anti-inflammatory properties. ${ }^{13,14}$ It is also effective in reducing levels of lipopolysaccharides (LPS)induced heart injury and decreasing malondialdehyde (MDA) levels, one of LPS-end products. ${ }^{13}$ Malondialdehyde (MDA) also has a potent role in inflammation, and it can increase the antioxidant glutathione (GSH) contents in heart tissue. ${ }^{13}$

Medical treatment with montelukast supplements was also beneficial in increasing the percentage of the EF among the montelukast-treated group as compareed to the Control group (Fig. 5). This is because montelukast has the ability of reducing the oxidative stress and apoptosis and providing beneficial effects on myocardial remodeling. . $^{15,16}$

This study also showed significant negative correlations between the ratio of $\mathrm{FEV}_{1} / \mathrm{FVC}$ and the IL6, TNF $\alpha$ (Figs 7 and 8), respectively. This proves the pulmonary protective effects of montelukast in reducing the inflammatory process and improving overall lung functions. ${ }^{17,18}$

\section{Conclusion}

This study shows the benefits of using pre-surgical montelukast supplement in ameliorating the inflammatory process in patients undergoing cardiopulmonary bypass during valve replacement surgery.

\section{Conflict of Interest}

Authors wish to declare that there is no conflict of interest, including specific financial interests and relationships and affiliations relevant to the subject of the manuscript, exist with this study.

\section{References}

1. Charbonney E, Wilcox E, Shan Y, d'Empaire PP, Duggal A, Rubenfeld GD, et al. Systemic angiopoietin-1/2 dysregulation following cardiopulmonary bypass in adults. Future Sci OA. 2017:3:FSO166.

2. Xie XJ, Tao KY, Tang ML, Du L, An Q, Lin K, et al. [Establishment and evaluation of extracorporeal circulation model in rats]. Sichuan Da Xue Xue Bao Yi Xue Ban. 2012;43:770-774.

3. Sablotzki A, Friedrich I, Muhling J, Dehne MG, Spillner J, Silber RE, et al. The systemic inflammatory response syndrome following cardiac surgery: different expression of proinflammatory cytokines and procalcitonin in patients with and without multiorgan dysfunctions. Perfusion. 2002;17:103-109.

4. Al-Rashid F, Kahlert P, Selge F, Hildebrandt H, Patsalis PC, Totzeck M, et al. Risk Assessment of patients undergoing transfemoral aortic valve implantation upon admission for post-interventional intensive care and surveillance: implications on short- and midterm outcomes. PloS one. 2016;11:e0167072.

5. Turagam MK, Mirza M, Werner PH, Sra J, Kress DC, Tajik AJ, et al. Circulating biomarkers predictive of postoperative atrial fibrillation. Cardiol Rev. 2016;24:76-87.

6. Zakkar M, Ascione R, James AF, Angelini GD, Suleiman MS. Inflammation, oxidative stress and postoperative atrial fibrillation in cardiac surgery. Pharmacol Ther. 2015;154:13-20.

7. Landis RC, Brown JR, Fitzgerald D, Likosky DS, Shore-Lesserson L, Baker RA, et al. Attenuating the systemic inflammatory response to adult cardiopulmonary bypass: a critical review of the evidence base. J Extra Corpor Technol. 2014:46:197-211.

8. Balani SK, Xu X, Pratha V, Koss MA, Amin RD, Dufresne C, et al. Metabolic profiles of montelukast sodium (Singulair), a potent cysteinyl leukotriene receptor antagonist, in human plasma and bile. Drug Metabol Dispos. 1997;25:1282-1287

9. Zhao JJ, Rogers JD, Holland SD, Larson P, Amin RD, Haesen R, et al. Pharmacokinetics and bioavailability of montelukast sodium (MK-0476) in healthy young and elderly volunteers. Biopharm Drug Dispos. 1997;18:769-777

10. Kuru S, Kismet K, Barlas AM, Tuncal S, Celepli P, Surer H, et al. The effect of montelukast on liver damage in an experimental obstructive jaundice model. Viszeralmedizin. 2015;31:131-138.

11. Peng J, Zhou H, Kuang G, Xie L, Tian T, Liu R. The selective cysteinyl leukotriene receptor 1 (CysLT1R) antagonist montelukast regulates extracellular matrix remodeling. Biochem Biophys Res Commun. 2017:484:474-479.

12. Said MM, Bosland MC. The anti-inflammatory effect of montelukast, a cysteinyl leukotriene receptor-1 antagonist, against estradiol-induced nonbacterial inflammation in the rat prostate. Naunyn Schmiedebergs Arch Pharmacol. 2017:390:197-205.

13. Khodir AE, Ghoneim HA, Rahim MA, Suddek GM. Montelukast attenuates lipopolysaccharide-induced cardiac injury in rats. Hum Exp Toxicol. 2016;35:388-397

14. Wang L, He Y, Zhang Y, Zhou H, Yu L, Yang J, et al. Effects of active components of Fuzi and Gancao compatibility on Bax, Bcl-2, and Caspase-3 in chronic heart failure rats. Evid Based Complement Alternat Med. 2016;2016:7686045

15. Becher UM, Ghanem A, Tiyerili V, Furst DO, Nickenig G, Mueller CF. Inhibition of leukotriene $\mathrm{C} 4$ action reduces oxidative stress and apoptosis in 
cardiomyocytes and impedes remodeling after myocardial injury. J Mol Cell Cardiol. 2011:50:570-577.

16. Mueller CF, Becher MU, Zimmer S, Wassmann S, Keuler B, Nickenig G. Angiotensin II triggers release of leukotriene $C 4$ in vascular smooth muscle cells via the multidrug resistance-related protein 1. Mol Cell Biochem. 2010;333:261-267.
17. Barbosa JS, Almeida Paz FA, Braga SS. Montelukast medicines of today and tomorrow: from molecular pharmaceutics to technological formulations. Drug Deliv. 2016;23:3257-3265.

18. Lajai N, llazi A, Kastrati B, Islami H. Comparison of glucocorticoid (budesonide) and antileukotriene (montelukast) effect in patients with bronchial asthma determined with body plethysmography. Acta Inform Med. 2015;23:347-351.

This work is licensed under a Creative Commons Attribution-NonCommercial 3.0 Unported License which allows users to read, copy, distribute and make derivative works for non-commercial purposes from the material, as long as the author of the original work is cited properly. 